IMA Journal of Applied Mathematics (2010) 75, 475-478

doi:10.1093/imamat/hxq024

\title{
Introduction to special issue on stability under finite deformation
}

\author{
MiCHEL DESTRADE* \\ School of Electric, Electronic and Mechanical Engineering, University College Dublin, \\ Belfield, Dublin 4, Ireland \\ *Corresponding author: destrade@1mm.jussieu.fr \\ AND \\ GIUSEPPE SACCOMANDI \\ Dipartimento di Ingegneria Industriale, Università degli Studi di Perugia, \\ 06125 Perugia, Italy
}

[Received on 15 March 2010; accepted on 18 March 2010]

In the botanical gardens of Grenoble, France, there is small bridge, just a few metres long, made of pre-stressed concrete. The bridge dates back to 1855 and is thought to be the oldest manufactured pre-stressed structure made with concrete. In general, a pre-stressed bridge span is reinforced by parallel metallic rods embedded below the horizontal mid-plane and put under tension, so that the slab is bent slightly upwards. When the bridge is put in place, the span is subjected to its own weight and to external loads, which ensure that the concrete is compressed everywhere. Without the prestress, the weight and loads would bend the span downwards, and create a zone of tension, of which concrete cannot sustain much. This technology thus combines the compressive strength of concrete with the tensile strength of the metallic rods. We can say that the resulting structure is stabilized by prestress.

Of course, a prestress or a prestrain can conversely lead to the destabilization of a solid. A classic example is that of the twisting instability of a closed ring: take an elastic straight rod, bend it into a circle by bringing the two ends together, but give the rod a twist before gluing its ends. For a sufficiently high twist, the elastic ring is unstable and folds unto itself, by forming an eight-shape curve (with about two turns of pretwist) or more elaborate shapes (for higher twists), see Fig. 1.

Stability studies are highly important for many engineering materials, be they subjected to small prestrains such as those imposed on pre-stressed concrete, as well as to finite prestrains, such as those imposed on elastomers in bridge bearings and engine mountings. These studies have developed into a fundamental topic of mathematical modelling because they are relevant not only to engineering problems but also to many biological, biomedical and biomechanical applications (DNA mechanics, cell stiffness, cellular structures, plant growth, microbial filaments, deformation of arteries and veins, skin wrinkling, etc.).

The discipline of elastic stability has a long and distinguished history, dating back at least to the works of Euler. For a comprehensive bibliography and an extensive treatment of most known problems, we refer, e.g. to the textbook by Bažant \& Cedolin (2003). For this introduction to the Special Issue on 'Stability under Finite Deformations', we chose to evoke three seminal papers, which we believe qualify as pioneering works in this field, and yet seem to be little known and forgotten (to the best of our knowledge). First, an article by Louis-Augustin Cauchy (1829), which contains the first derivation of the equations of motion in a solid which is already in a state of stress. According to Truesdell (1966), Cauchy's 'results were not understood and were reported obscurely or even incorrectly by 19th century

(C) The Author 2010. Published by Oxford University Press on behalf of the Institute of Mathematics and its Applications. All rights reserved. 

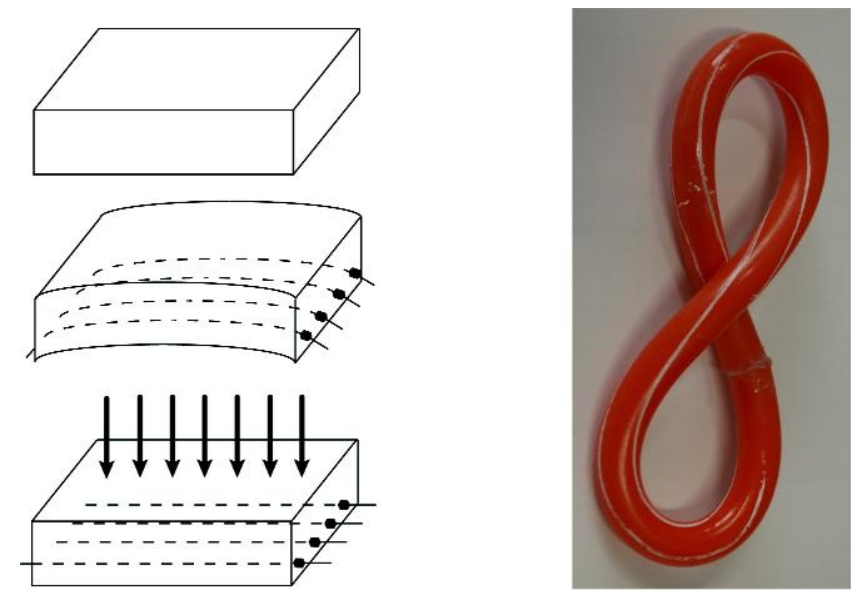

FIG. 1. On the left: Stabilizing a bridge span made of concrete by reinforcing it with steel rods put under tension. Once the span is put in place and subject to loads, the concrete is compressed everywhere. On the right: Destabilizing a ring by twisting a polyurethane rod before gluing its two ends together. For a large enough pretwist, the ring folds unto itself in an eight-shape.

expositors'. By the time Rayleigh (1906) modelled the Earth as a solid with initial stress, 'Cauchy's results had long been forgotten', according to Man \& Lu (1987). Second, a most elegant four-page treatment of the twisted elastic ring instability by Michell (1889-1890). According to Goriely (2006), this Australian applied mathematician cuts an 'almost tragic figure', whose work has only recently begun to be fully appreciated. In particular, Goriely (2006) found out that Michell's result on ring instability has been rediscovered at least three times in the 20th century. Third, a 1940 article by the Belgian applied mathematician, Maurice Anthony Biot (1940). Although Biot is well remembered for laying down the foundations of the theory of poroelasticity, it is not so well acknowledged that he can be credited with the modern derivation of the incremental equations of non-linear elasticity (his results are beautifully synthesized in his 1965 monograph, now out-of-print (Biot, 1965)). In his 1940 article in the Journal of Applied Physics, he gave a simple and direct exposition of the incremental equations of elastodynamics and applied it to the study of the influence of initial stress on seismic waves. It is now widely accepted that there is a deep connection between wave motion and stability. Jacques Hadamard established a theorem about this connection for a general elastic medium as early as 1903 . We may summarize crudely the Hadamard theorem ${ }^{1}$ by stating that if a linear elastic system is stable, then wave propagation is possible in every direction. The 1940 Biot paper is the modern delineation of Hadamard's theorem and proposes a general method of studying the stability of pre-stressed solids by considering small-amplitude (or incremental) motions of the system in the neighbourhood of a large deformation equilibrium.

We are now several decades after the Hadamard theorem and the seminal investigation by Biot, and the connection between stability and incremental motions and deformations is well established. In the 1960s, several important papers on this subject were written by Hayes, Hill, Rivlin, Toupin and many others. The first fundamental summary on elastic stability appeared in the Handbuch der Physik, by Knops \& Wilkes (1972). A continuous interest in the subject can be recorded through the subsequent

\footnotetext{
${ }^{1}$ The original proof of this theorem (Hadamard, 1903) seems to be flawed, as pointed out by Duhem. In any case, Gaetano Fichera and Carlo Cattaneo were later able to fix the proof, see, e.g. Cattaneo (1972).
} 
years, and today many problems involving not only incremental but also small-but-finite motions and deformations of an elastic system in the neighbourhood of a non-linear finite deformation have been completely resolved. New tools and algorithms have been developed to handle complex situations and solve highly non-linear problems.

Despite these developments, we have to acknowledge that the study of stability issues in solid mechanics is still in a status of underdevelopment with respect to what has been done and achieved in fluid mechanics. The aim of this Special Issue is to display some of the current advances in this field and we have indeed been fortunate to obtain nine outstanding papers.

Two of these papers are concerned with classical stability problems of non-linear elasticity theory. Biscari \& Omati study the so-called Knowles material, a well-used constitutive model of finite elasticity, able to account for shear stiffening or shear softening by the adjustment of a single parameter. By widening the range of acceptable values for the material parameters, the authors show that the Knowles model encompasses a wide array of generalized neo-Hookean materials, including the popular Gent and Fung models. Hence, several problems of non-linear elasticity can be solved under the same umbrella, as the authors demonstrate with their study in the stability of a thin spherical shell under large inflation. Pucci \& Saccomandi use the framework of non-linear viscoelasticity of differential type to show that finite-amplitude shearing motions superimposed on an unsteady simple extension are admissible motions. The amplitude of the shear motions may then be determined by solving a non-linear non-autonomous differential equation for which limit cycles are possible. The authors then relate their results to the classic Melde string experiment of parametric resonance.

This Special Issue also proposes two expert treatments of some non-linear stability problems in structural mechanics. Wang \& Dai study the bifurcations of the uni-axial compression of an incompressible 2D rectangular block, with welded boundary conditions. Via a series-asymptotic expansion method, they determine two coupled non-linear ordinary differential equations governing the leading order of the axial strain and shear strain. Then they obtain the critical stress values for buckling of the plate and the post-bifurcation solutions for the first three modes. Roccabianca, Gei \& Bigoni solve explicitly the finite-plane strain bending problem for a multi-layered incompressible thick plate. Their bending solution is then employed to investigate possible incremental bifurcations. The analysis reveals that a multi-layered structure can behave in a completely different way from a homogeneous plate. The authors also compare their theoretical predictions with very revealing experimental data.

Three papers are devoted to stability issues of non-linear solid biomechanics. Goriely \& Vandiver are interested in arterial wall mechanics. They model arteries as incompressible two-layer cylindrical structures that are residually stressed through differential growth. When these structures are loaded by an axial force and internal pressure, a buckling instability is possible, which may eventually lead to arterial tortuosity. The authors also investigate the potential role of axial residual stress in regulating stress in arteries and preventing such buckling instabilities. Dervaux \& Ben Amar examine the structural heterogeneity of stratified media that lose their initial symmetry when they grow. Mismatching expansions between distinct layers act as a geometrical constraint that leads to the emergence of wavy patterns. This instability may explain the formation of biological patterns, such as fingerprints. Studying a locally growing thin sheet bound to a soft semi-infinite substrate, the authors show that in two dimensions, bending effects select the shape of the growing sheet, therefore preventing the fabrication of an arbitrary surface. Pearce \& Fu consider the localized bulging/necking of an inflated hyperelastic membrane tube with closed ends, a problem that may be relevant to the modelling of aortic aneurysm formation. The initiation pressure for the onset of localized bulging is simply the limiting pressure in uniform inflation when the axial force is held fixed. It is then possible to compute analytically how, as 
inflation continues, the initial bulge grows continually in diameter until it reaches a critical size and then propagates in both directions. The authors also study the stability of the solutions with respect to axially symmetric perturbations, by using the compound matrix method to compute the Evans function.

Finally, two papers fall into the realm of multi-physics and coupled field theories. Dorfmann \& Ogden devote a paper to the mechanics of electroactive elastomers, which are capable of large deformations when subject to an applied electrical field. They study the propagation of small-amplitude electromechanical waves in a non-linear electroelastic material subject to a bias in the quasi-electrostatic approximation. The authors derive the general incremental equations and apply them to the study of surface waves in a homogeneously deformed half-space of incompressible isotropic material with an applied static electric field normal to the surface. They then make the link with surface stability issues. In the paper by Liu \& Quintanilla, it is possible to appreciate how some modern mathematical tools can be used to study stability. The authors consider the system of governing equations for homogeneous, isotropic, pre-stressed elastic plates, within a particular theory of thermo-elasticity. Using the theory of semigroups of differential operators, they show that the solutions for this model must be analytic, a theorem which is fundamental to the establishment of general stability results.

We hope that the readers will enjoy this collection of papers and will feel inspired. Many important and interesting problems remain to be solved in this field, and a lot of work is still required in order to establish complete and rigorous mathematical results. Nonetheless, things are moving at a fast pace, and beautiful and interesting results are coming through.

We thank the Editorial Board and the editorial staff of the IMA Journal of Applied Mathematics for the professional assistance during the realization of this Special Issue, especially our good friend Yibin Fu.

\section{REFERENCES}

BAŽAnt, Z. P. \& Cedolin, L. (2003) Stability of Structures: Elastic, Inelastic, Fracture, and Damage Theories. New York: Dover.

Bıот, M. A. (1940) The influence of initial stress on elastic waves. J. Appl. Phys., 11, 522-530.

Biot, M. A. (1965) Mechanics of Incremental Deformations. Theory of Elasticity and Viscoelasticity of Initially Stressed Solids and Fluids, Including Thermodynamic Foundations and Applications to Finite Strain. New York: John Wiley.

Cattaneo, C. (1972) Waves and stability. J. Elast., 2, 91-99.

CAUChy, A.-L. (1829) Sur l'équilibre et le mouvement intérieur des corps considérés comme des masses continues. Exerc. Math., 4, 293-319. Reprinted, pp. 342-369 of Oeuvres Complètes d'Augustin Cauchy, série II, tome IX, Gauthier-Villars, Paris, 1891. Reprint available at http://mathdoc.emath.fr/cgi-bin/oetoc?id= OE_CAUCHY_2_9.

Goriely, A. (2006) Twisted elastic rings and the rediscoveries of Michell's instability. J. Elast., 84, 281-299.

HADAMARD, J. (1903) Lecons sur la propagation des ondes et les équations de l'hydrodynamique. Paris: Hermann.

KNOPS, R. J. \& WILKES, E. W. (1972) Theory of elastic stability. Handbuch der Physik, vol. VI/a3, (C. Truesdell ed.). Berlin: Springer.

MAN, C.-S. \& LU, W. Y. (1987) Towards an acoustoelastic theory for measurement of residual stress. J. Elast., 17, 159-182.

MiCheLl, J. H. (1889-1890) On the stability of a bent and twisted wire. Messenger Math., 11, 181-184.

RAYLEIGH, L. (1906) On the dilatational stability of the earth. Proc. R. Soc. Lond. A, 77, 486-499.

Truesdell, C. (1966) The Mechanical Foundations of Elasticity and Fluid Dynamics. New York: Gordon and Breach. 